\title{
A method for small-animal PET/CT alignment calibration
}

\author{
J Pascau $^{1,2}$, J J Vaquero ${ }^{1,2}$, J Chamorro-Servent ${ }^{2}$, A Rodríguez-Ruano ${ }^{1}$ \\ and $M \operatorname{Desco}^{1,2}$ \\ ${ }^{1}$ Unidad de Medicina y Cirugía Experimental, Hospital General Universitario Gregorio \\ Marañón, Madrid, Spain \\ 2 Departamento de Bioingeniería e Ingeniería Aeroespacial, Universidad Carlos III de Madrid, \\ Madrid, Spain \\ E-mail: javier.pascau@uc3m.es
}

\begin{abstract}
Small-animal positron-emission tomography/computed tomography (PET/ CT) scanners provide anatomical and molecular imaging, which enables the joint visualization and analysis of both types of data. A proper alignment calibration procedure is essential for small-animal imaging since resolution is much higher than that in human devices. This work presents an alignment phantom and two different calibration methods that provide a reliable and repeatable measurement of the spatial geometrical alignment between the PET and the CT subsystems of a hybrid scanner. The phantom can be built using laboratory materials, and it is meant to estimate the rigid spatial transformation that aligns both modalities. It consists of three glass capillaries filled with a positron-emitter solution and positioned in a non-coplanar triangular geometry inside the system field of view. The calibration methods proposed are both based on automatic line detection, but with different approaches to calculate the transformation of the lines between both modalities. Our results show an average accuracy of the alignment estimation of $0.39 \mathrm{~mm}$ over the whole field of view.
\end{abstract}

\section{Introduction}

Small-animal positron-emission tomography/computed tomography (PET/CT) scanners provide joint anatomical and molecular imaging, making it possible the combined visualization and analysis of both modalities, PET attenuation correction (Kinahan et al 1998) and even an increase in diagnostic accuracy (Dong et al 2008).

Several software registration techniques have been developed to match images from different modalities (Slomka and Baum 2009), while hardware-based procedures claim to 

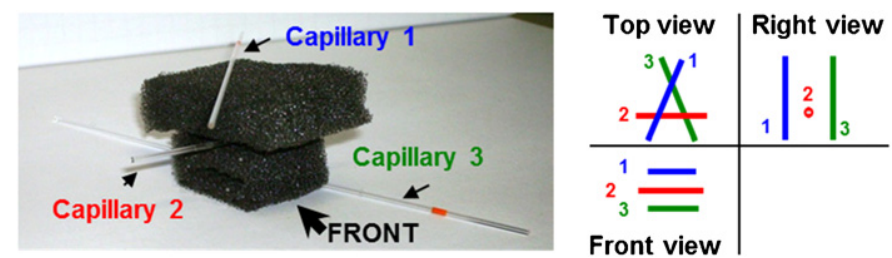

Figure 1. Example of the proposed phantom showing the three capillaries separated by foam material (left) and the capillaries location, depicted as three projections on orthogonal planes (right).

ensure the repeatability of the alignment independently of the image contents. In the case of small-animal imaging, specific holders have been proposed to facilitate this process by using external references (Chow et al 2006, Kokuryo et al 2010, Woo et al 2009, Suckow et al 2009). Another alternative is combining CT and PET in a single hybrid device, an option that presents a number of advantages (Townsend et al 2004). In these devices, the two axially displaced fields of view (FOV) can be mechanically aligned only to a certain extent, and the residual errors can impair sub-millimetric registrations. Since the intrinsic spatial resolution of these small-animal systems is much higher than that of human scanners, high resolution fused studies require more precise registration of anatomical and functional imaging data (Vaquero et al 2001). Therefore, PET/CT systems for small-animal imaging involve a calibration procedure to accurately calculate and correct these residual misalignments, and it is desirable that this procedure could be easily replicated for quality assurance purposes.

The alignment of PET/CT scanners usually assumes a rigid-body transformation in order to match the images from both modalities. Affine or elastic transformations are not required if the sample is not manipulated between scans and both subsystems have been geometrically calibrated in advance. The challenge in aligning PET/CT systems is to design a simple phantom that enables reliable misalignment calculations, with low computational cost and no user dependence. It should also be suitable for alignment calibration of dual modality systems in an end-user environment, as these systems need to be calibrated on a regular basis (Gregory et al 2006).

This work presents an alignment phantom and two different registration methods that lead to a reliable and repeatable measurement of the geometrical relationship between PET and CT subsystems. We have designed a three-dimensional phantom that can be built with standard laboratory materials. Both registration methods used are based on automatic line detection to calculate the rigid alignment: One of them localizes pairs of correspondent points within the lines in both modalities, while the other is an iterative method that minimizes the distance between line sets, using angle and position information. We also present validation experiments to determine if the methods are accurate, stable and reliable.

\section{Materials and methods}

\subsection{Phantom description}

The proposed phantom consists of three non-coplanar glass capillaries filled with a positronemitter solution (FDG in our case), thus being visible in both modalities. These capillaries are arranged on a triangular geometry in the plane view, but each one is located at a different height (figure 1). The capillaries used are micropipettes (BLAUBRAND intraMARK Cat no 708709 with $10 \mu \mathrm{l} / 1.24 \mathrm{~mm}$ external diameter), separated by a low density foam 


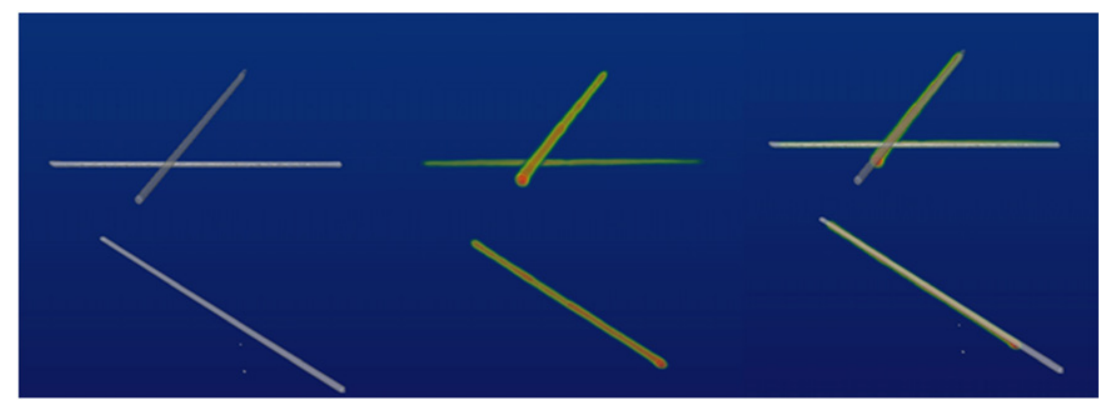

Figure 2. CT (left), PET (middle) and PET/CT fusion (right) render views of one of the phantoms, showing the three capillaries clearly visible on both modalities.

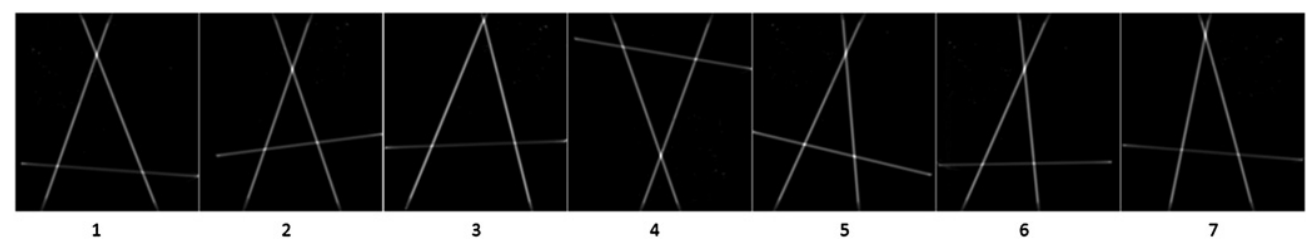

Figure 3. Top view, as described in figure 1, of the seven phantom configurations used to test the repeatability of the proposed methodology.

material, thus achieving a large density difference between the glass capillaries and the background material.

\subsection{Image acquisition}

A small-animal PET/CT system (Argus/CT, Suinsa Medical Systems) was used for the acquisition of CT and PET images (Vaquero et al 2008, Wang et al 2006). Each CT scan was reconstructed using a specific FDK-based software (Abella et al 2011) into CT datasets with matrix size $256 \times 256 \times 256 \mathrm{~mm}^{3}$ and voxel size $0.123 \times 0.123 \times 0.123 \mathrm{~mm}^{3}$. PET scan was reconstructed using the 2D-OSEM algorithm available in the Argus scanner into $175 \times$ $175 \times 61 \mathrm{~mm}^{3}$ matrix size datasets with a voxel size of $0.3875 \times 0.3875 \times 0.775 \mathrm{~mm}^{3}$. In order to simulate variability under real conditions in a laboratory environment, seven phantoms were built with different geometrical configurations of the capillaries (angles and distances). All phantoms were acquired with the PET/CT system and the proposed methods were tested on all of them, in order to assess the repeatability of the results. Phantoms were injected with residual dose at the end of the day. Acquisition time was decided depending on the observed coincidence rate to achieve a total count number ranging from 1.7 to 3.9 million. Figure 2 shows a render view of one of the phantoms on both modalities, and the fused datasets. Figure 3 shows top views of the seven phantoms.

\subsection{Registration methods}

We have tested two different registration methods to obtain the 3D rigid transformation (including only translations and rotations) that matches PET/CT image pairs. Despite following different strategies, both methods require a common initial step based on line 
detection, on which images from both modalities are reduced to two line sets. From the equations of these lines, the first registration method calculates the transformation parameters by identifying pairs of homologous points, while the second iteratively minimizes the distance between both line sets.

2.3.1. Common step: background thresholding, region labeling and line detection. After CT and PET phantom images have been acquired, the first step is to detect the lines that correspond to each of the capillaries. First, the images are automatically reduced to point sets that correspond to the capillaries by thresholding the background and labeling the regions that correspond to the capillaries as follows:

$$
\begin{aligned}
& \left.X_{n}=\text { [points on CT image corresponding to capillary } n\right], \\
& \left.Y_{n}=\text { [points on PET image corresponding to capillary } n\right], \\
& n=1,2,3 .
\end{aligned}
$$

The threshold value was constant for all the CT images (2500 HU), while for the PET images the expected total volume of the three capillaries was used to determine the threshold. Knowing that the capillaries appear in the PET image with larger apparent diameter than the real one due to the PET spillover, all pixels inside 1.65 times the real diameter of the capillaries will include approximately $580 \mathrm{~mm}^{3}$. This value allowed calculating an automatic threshold for every PET image, in such a way that we included pixels with highest intensities up to reaching that volume (5000 pixels for our PET pixel size), and the rest were considered as background. The resulting binary images were then labeled to obtain the three largest regions (the labeling process searches for independent groups of connected pixels), and every cluster of pixels was then classified as capillary ' $n$ ' depending on its relative $z$ position in the image (as depicted in the frontal view in figure 1). Afterward, we identified the lines that describe the position and orientation of each of the capillaries by means of principal component analysis applied to each distribution of points $X_{n}$ and $Y_{n}$ (Duda et al 2000). Given a cloud of points, the direction of the line corresponds to the eigenvector associated with the maximum eigenvalue of their covariance matrix, while the line position is defined by the centroid of the point set.

Here, $X_{n}=\left(a_{n}, \mathbf{u}_{\mathbf{n}}\right)$ is the line related to capillary $n$ on the CT image, represented by its position $a_{n}$ and its direction vector $\mathbf{u}_{n}$. Similarly, in the PET image, $Y_{n}=\left(b_{n}, \mathbf{v}_{n}\right)$.

At this point, each dataset has been reduced to three line equations, and from here on, each of the two methods will extract different information from the line sets in order to align them.

2.3.2. Method 1. This method obtains the best matching between both line sets by identifying and aligning pairs of homologous points. The procedure to define these corresponding points is based on locating the closest point from one line to another within the line set, which are uniquely defined in both modalities. For instance, the closest point on capillary 1 to capillary 2 on the CT image corresponds with the closest point on capillary 1 to capillary 2 on the PET image. The common perpendicular between two lines defines the closest points between those lines (figure 4). This is equivalent to solving the following equation system for each pair of lines:

$$
\left\{\begin{array}{l}
a_{1 x}-a_{2 x}=-\mu \cdot u_{1 x}+\lambda \cdot u_{2 x}+t \cdot w_{x}, \\
a_{1 y}-a_{2 y}=-\mu \cdot u_{1 y}+\lambda \cdot u_{2 y}+t \cdot w_{y}, \\
a_{1 z}-a_{2 z}=-\mu \cdot u_{1 z}+\lambda \cdot u_{2 z}+t \cdot w_{z},
\end{array}\right.
$$

where lines are defined by one point $\left(a_{n x}, a_{n y}, a_{n z}\right)$ and one vector $\left(u_{n x}, u_{n y}, u_{n z}\right), \mathbf{w}=\mathbf{u}_{2} \times \mathbf{u}_{1}$. After solving the system, $\mu, \lambda$ and $t$ allow us to calculate the points as $R=a_{1}+\mu \cdot \mathbf{u}_{1}$ and $S=a_{2}+\lambda \cdot \mathbf{u}_{2}$. 

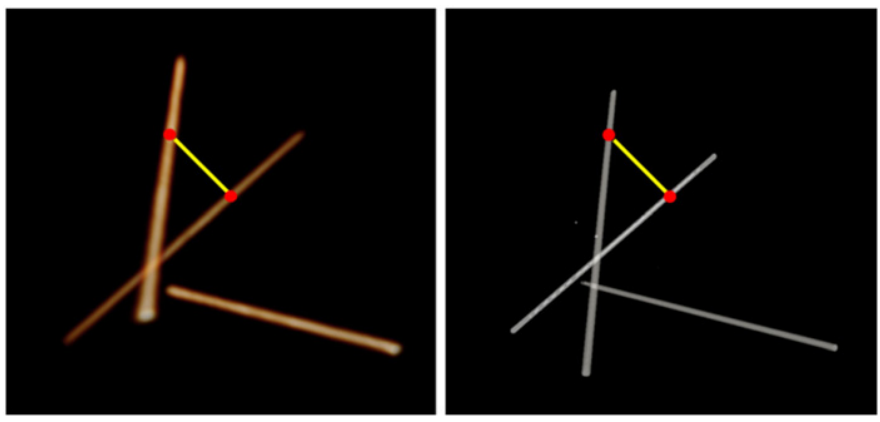

Figure 4. For every two images in each dataset, the common perpendicular (yellow line) intersects at two points (red dots) that are homologous in both datasets (CT on the left and PET on the right).

The result of this process is a set of six pairs of homologous landmarks. Since these landmarks define a point-to-point correspondence, they can be used to calculate the transformation that aligns the images by solving the least-squares problem using the approach proposed in Arun et al (1987). The final PET/CT alignment parameters (translation and rotation) can be derived from the transformation matrix obtained.

2.3.3. Method 2. The rationale of this method is to find the rigid transformation that yields the best match between both line sets, in terms of a cost function that depends on distance and orientation. Matching two sets of corresponding 3D lines is a basic tool in computer vision that has applications in different fields (Daniilidis, 1999, Kamgar-Parsi 2004, Zhang and Faugeras 1991). The first step is to determine line to line correspondences, which is a minor problem in our case. However, determining the corresponding portions within lines is a non-trivial problem, since both datasets do not have the same FOV. In this work, we have used a closed-form algorithm developed by Kamgar-Parsi (2004), which can be summarized as follows.

The distance between two lines $D\left(X_{n}, Y_{n}\right)$ is defined as the sum of the distances between all pairs of corresponding points. Supposing that $a_{n}$ and $b_{n}+s_{n} \mathbf{v}_{\mathbf{n}}$ is a pair of corresponding points:

$$
D\left(X_{n}, Y_{n}\right)=\int_{\Omega_{n}} \operatorname{dist}\left(a_{n}+\alpha \mathbf{u}_{\mathbf{n}}, b_{n}+s_{n} \mathbf{v}_{\mathbf{n}}+\alpha_{n} \mathbf{v}_{\mathbf{n}}\right) \cdot \mathrm{d} \alpha,
$$

where $\alpha$ is a scalar variable that parameterizes the lines, $\Omega_{n}$ is the overlap between $X_{n}$ and $Y_{n}$, 'dist' is the distance function between points, and $s_{n}$ is the shift parameter that specifies the portion of $Y_{n}$ that corresponds to the portion of $X_{n}$. With $T$ being the transformation matrix, the distance between both sets can be expressed as

$$
M(X, T Y)=\sum_{n=1}^{N} D\left(X_{n}, T Y_{n}\right) .
$$

Using the sum of the squared Euclidean distance of corresponding pairs $\left(£_{2}\right.$ norm $)$ as a distance function and decomposing $T$ into a rotation matrix $(R)$ and a translation vector $(t)$, the equation becomes:

$$
\begin{aligned}
M(X, \mathrm{~T} Y) & =\sum_{n=1}^{3} \int_{-L_{n} / 2}^{L_{n} / 2}\left\|\left(a_{n}-t-R\left(b_{n}+s_{n} \mathbf{v}_{\mathbf{n}}\right)\right)+\alpha\left(\mathbf{u}_{\mathbf{n}}-R \mathbf{v}_{\mathbf{n}}\right)\right\|^{2} \partial \alpha \\
& =\sum_{n=1}^{3}\left[L_{n}\left\|a_{n}-t-R\left(b_{n}+s_{n} \mathbf{v}_{\mathbf{n}}\right)\right\|^{2}+\frac{L_{n}^{3}}{6}\left(1-\mathbf{u}_{\mathbf{n}}^{\mathbf{T}} R \mathbf{v}_{\mathbf{n}}\right)\right],
\end{aligned}
$$


where $X_{n}$ and $Y_{n}$ are supposed to have equal length $L_{n}$. In our case, $L_{n}$ was estimated as the minimum length of the capillary segments visualized within the FOV in both modalities.

The best match between the two sets is finally obtained by minimizing $M(X, T Y)$ over all the possible transformations $T$ and shift values $s_{n}$ using a least-squares approach (initial value for $s_{n}=\{0\}$ ). The rotation matrix, translation vector and shift parameter are calculated at each iteration. We used the singular value decomposition of the cross-covariance matrix to compute the rotation matrix. The convergence criterion was a difference between $s_{n}$ values in consecutive iterations below a certain heuristic threshold $\left(10^{-3}\right)$, which leads to less than 20 shifts in all cases.

\subsection{Validation}

With the purpose of evaluating the alignment accuracy achieved by each method, we used two different measurements to estimate the registration error at the center and corners of the FOV, respectively.

The calibration phantom was scanned together with an additional encapsulated ${ }^{22} \mathrm{Na}$ point source placed near the center of the FOV, clearly visible in both modalities. This point was neglected during the registration process and was only used to assess the error at the center of the FOV, by measuring the distance between the center of the transformed marker in the PET $\left(\mathrm{PET}_{\mathrm{POS}}\right)$ and $\mathrm{CT}\left(\mathrm{CT}_{\mathrm{POS}}\right)$ datasets:

$$
\text { Error }=\operatorname{Norm}\left(\hat{R} \times \mathrm{PET}_{\mathrm{pos}}+\hat{T}-\mathrm{CT}_{\mathrm{pos}}\right),
$$

where $\hat{R}$ and $\hat{T}$ are the estimated rotation and the translation.

In order to assess the registration error at the corners of the FOV, we acquired a special phantom consisting of an approximated right prism foam structure with eight landmarks located at the vertices. The approximated sizes of the sides were 40, 20 and $40 \mathrm{~mm}$ along $x, y$ and $z$ directions. These landmarks were pumice stone fragments smaller than $1 \mathrm{~mm}$, dipped in FDG solution and, thus, visible in both modalities. This phantom was built and imaged three times following this description, but with small changes on the distribution of the landmarks. These differences allow us to check the repeatability of the procedure.

\subsection{Fiducial localization protocol}

The coordinates of the ${ }^{22} \mathrm{Na}$ point sources and the pumice stone fragments required for the validation were obtained with a semi-automatic approach to avoid user-related errors. Every marker was first identified by a user in the CT or the PET image. The center of mass of the marker was then calculated from all pixels connected to that position and with an intensity value within a certain range. That intensity interval was the same for all CT images: from $500 \mathrm{HU}$ to the maximum CT image value. For the PET images, the maximum was set as the image intensity in the initial marker position (the one selected by the user) and the minimum as $70 \%$ of that value.

\section{Results}

Computation time was below $5 \mathrm{~s}$ for both registration methods (running on an Intel(R) Core(TM) 2 Quad CPU, $2.39 \mathrm{GHz}$ with 4 GB RAM Memory, under Windows XP x64 Operating System).

The error at FOV center was measured for every method with the ${ }^{22} \mathrm{Na}$ point source as described above. The average mean-squared error at that point was 0.276 and $0.346 \mathrm{~mm}$, for methods 1 and 2 , respectively (see table 1 ). 
Table 1. Error at the center of the FOV ( $\mathrm{mm}$ ) of both methods with different geometric configuration of the phantom.

\begin{tabular}{lll}
\hline & Method 1 & Method 2 \\
\hline Configuration 1 & 0.353 & 0.417 \\
Configuration 2 & 0.211 & 0.403 \\
Configuration 3 & 0.139 & 0.178 \\
Configuration 4 & 0.190 & 0.413 \\
Configuration 5 & 0.438 & 0.419 \\
Configuration 6 & 0.280 & 0.225 \\
Configuration 7 & 0.319 & 0.366 \\
Mean & 0.276 & 0.346 \\
Standard deviation & 0.104 & 0.101 \\
\hline
\end{tabular}

Table 2. Average error at the corners of the FOV $(\mathrm{mm})$ of both methods with different geometric configuration of the pumice phantom, which was built three times to evaluate repeatability.

\begin{tabular}{|c|c|c|c|c|c|c|}
\hline & \multicolumn{2}{|c|}{ Pumice phantom 1} & \multicolumn{2}{|c|}{ Pumice phantom 2} & \multicolumn{2}{|c|}{ Pumice phantom 3} \\
\hline & Method 1 & Method 2 & Method 1 & Method 2 & Method 1 & Method 2 \\
\hline Configuration 1 & 0.248 & 0.268 & 0.362 & 0.367 & 0.296 & 0.304 \\
\hline Configuration 2 & 0.540 & 0.370 & 0.513 & 0.397 & 0.512 & 0.384 \\
\hline Configuration 3 & 0.322 & 0.324 & 0.378 & 0.385 & 0.346 & 0.359 \\
\hline Configuration 4 & 0.333 & 0.308 & 0.416 & 0.394 & 0.355 & 0.347 \\
\hline Configuration 5 & 0.290 & 0.291 & 0.378 & 0.379 & 0.311 & 0.313 \\
\hline Configuration 6 & 0.435 & 0.443 & 0.501 & 0.511 & 0.497 & 0.502 \\
\hline Configuration 7 & 0.422 & 0.433 & 0.516 & 0.517 & 0.451 & 0.439 \\
\hline Mean & 0.370 & 0.348 & 0.438 & 0.422 & 0.396 & 0.378 \\
\hline Standard deviation & 0.101 & 0.069 & 0.070 & 0.064 & 0.089 & 0.071 \\
\hline
\end{tabular}

The same transformations were also applied to align the pumice phantom acquisitions, as depicted in section 2.4. The average errors at the eight vertices are shown in table 2. No statistically significant differences were found between these values for both methods using an Anova test $(p>0.05)$ with one fixed factor (method) and two random factors (configuration and phantom); Levene's test was used to check homoscedasticity and the Kolmogorov-Smirnov test was used to check for normality.

\section{Discussion and conclusions}

Our results demonstrate that the algorithms and phantom presented here are suitable to estimate the PET/CT alignment in a dual-modality system, with small registration error. The design of the phantom has also shown to be simple and robust, since constructive differences did not affect the alignment accuracy. Although the error values change depending on the configuration, these differences are very small compared to the PET resolution and are probably related to the mechanical accuracy of the whole system. The results for three pumice phantoms (average alignment error $0.39 \pm 0.08 \mathrm{~mm}$ ) demonstrate the robustness of the alignment with both methods and are representative of the real registration quality in the whole image.

Methods to address this alignment problem using landmark distributions along the FOV have been described (Gregory et al 2006, Liang et al 2007, Vaquero et al 2007). The main disadvantage of these approaches is that the user must manually place the point sources uniformly throughout the FOV in order to obtain good registration accuracy in the area of 
interest. Chow et al (2006) presented a three-dimensional phantom with 1288 lines, admitting that building the phantom was not an easy task. $\mathrm{Ng}$ et al (2010) employed a rod phantom with semi-automated alignment for a PET/MR scanner, measuring the registration error only at the phantom centroid. Jan et al (2006) used a three-line-source phantom, where they analyzed 13 slices from both modalities to iteratively calculate the registration. Recently, Feng et al (2011) presented a similar approach for small animal PET/CT, which makes use of a prebuilt phantom with four ${ }^{22} \mathrm{Na}$ point sources. The registration error in that case (from $0.44 \pm 0.18$ to $0.33 \pm 0.11 \mathrm{~mm}$ ) is in the same range as our results. The main advantage of our method is that it is based on an extremely simple calibration phantom that users can easily build on their own with standard laboratory materials.

Validation of the registration algorithms presented in this paper demonstrates that both of them are suitable to properly match two sets of corresponding 3D lines and are valid to estimate the transformation between the FOVs. The proposed methodology is reliable for PET/CT alignment calibration of small-animal dual modality systems in an end-user environment, achieving high accuracy with low computational cost and a simple and affordable phantom.

\section{Acknowledgments}

This study was funded by CDTI under the CENIT Program (AMIT Project), projects ARTEMIS S2009/DPI-1802 (CAM) and TEC2010-21619-C04-01, and supported by the Spanish Ministry of Economy and Competitiveness.

\section{References}

Abella M, Vaquero J J, Sisniega A., Pascau J., Udías A, García V, Vidal I and Desco M 2011 Software architecture for multi-bed FDK-based reconstruction in x-ray CT scanners Comput. Methods Programs Biomed. at press (Available online at http://dx.doi.org/10.1016/j.cmpb.2011.06.008)

Arun K S, Huang T S and Blostein S D 1987 Least-squares fitting of two 3D point sets IEEE Trans. Pattern Anal. Mach. Intell. 9 698-700

Chow P L, Stout D B, Komisopoulou E and Chatziioannou A F 2006 A method of image registration for small animal, multi-modality imaging Phys. Med. Biol. 51 379-90

Daniilidis K 1999 Hand-eye calibration using dual quaternions Int. J. Robot. Res. 18 286-98

Dong M J, Zhao K, Lin X T, Zhao J, Ruan L X and Liu ZF 2008 Role of fluorodeoxyglucose-PET versus fluorodeoxyglucose-PET/computed tomography in detection of unknown primary tumor: a meta-analysis of the literature Nucl. Med. Commun. 29 791-802

Duda R, Hart P and Stork D 2000 Pattern Classification 2nd edn (New York: Wiley-Interscience)

Feng B, Yan S, Chen M, Austin D W, Deng J and Mintzer R A 2011 Automated least-squares calibration of the coregistration parameters for a micro PET-CT system IEEE Trans. Nucl. Sci. 58 2303-7

Gregory R, Partridge M and Flower M A 2006 Performance evaluation of the Philips "Gemini" PET/CT system IEEE Trans. Nucl. Sci. 53 93-101

Jan M-L, Ni Y-C, Chen K-W, Liang H-C, Chuang K-S and Fu Y-K 2006 A combined micro-PET/CT scanner for small animal imaging Nucl. Instrum. Methods Phys. Res. A 569 314-8

Kamgar-Parsi B 2004 Algorithms for matching 3D line sets IEEE Trans. Pattern Anal. Mach. Intell. 26 582-93

Kinahan P E, Townsend D W, Beyer T and Sashin D 1998 Attenuation correction for a combined 3D PET/CT scanner Med. Phys. 25 2046-53

Kokuryo D, Kimura Y, Obata T, Yamaya T, Kawamura K, Zhang M R, Kanno I and Aoki I 2010 A small animal holding fixture system with positional reproducibility for longitudinal multimodal imaging Phys. Med. Biol. 55 4119-30

Liang H, Yang Y, Yang K, Wu Y, Boone J M and Cherry S R 2007 A microPET/CT system for in vivo small animal imaging Phys. Med. Biol. 52 3881-94

Ng T S C, Procissi D, Wu Y and Jacobs R E 2010 A robust coregistration method for in vivo studies using a first generation simultaneous PET/MR scanner Med. Phys. 37 1995-2003

Slomka P J and Baum R P 2009 Multimodality image registration with software: state-of-the-art Eur. J. Nucl. Med. Mol. Imaging 36 (Suppl. 1) S44-55 
Suckow C, Kuntner C, Chow P, Silverman R, Chatziioannou A and Stout D 2009 Multimodality rodent imaging chambers for use under barrier conditions with gas anesthesia Mol. Imaging Biol. 11 100-6

Townsend D W, Carney J P, Yap J T and Hall N C 2004 PET/CT today and tomorrow J. Nucl. Med. 45 (Suppl. 1) S4-S14 (Available at http://jnm.snmjournals.org/content/45/1_suppl/4S.abstract)

Vaquero J J, Desco M, Pascau J, Santos A, Lee I, Seidel J and Green M V 2001 PET, CT, and MR image registration of the rat brain and skull IEEE Trans. Nucl. Sci. 48 1440-5

Vaquero J J, Pascau J, Abella M, Sisniega A, Lage E and Desco M 2007 Field of view alignment on a multimodality PET/CT scanner for small animals IEEE Nuclear Science Symp. Conf. Record 4 2868-9

Vaquero J J, Redondo S, Lage E, Abella M, Sisniega A, Tapias G, Soto-Montenegro M L and Desco M 2008 Assessment of a new high-performance small-animal x-ray tomograph IEEE Trans. Nucl. Sci. 55 898-905

Wang Y, Seidel J, Tsui B M W, Vaquero J J and Pomper M G 2006 Performance evaluation of the GE Healthcare eXplore VISTA dual-ring small-animal PET scanner J. Nucl. Med. 47 1891-900 (Available at http://jnm.snmjournals.org/content/47/11/1891.abstract)

Woo S K, Kim K M, Lee T S, Jung J H, Kim J G, Kim J S, Choi T H, Il An G and Cheon G J 2009 Registration method for the detection of tumors in lung and liver using multimodal small animal imaging IEEE Trans. Nucl. Sci. 56 1454-8

Zhang Z Y and Faugeras O D 1991 Determining motion from 3D line segment matches - a comparative-study Image Vis. Comput. 9 10-19 